\title{
Vessicular Glutamate Transporters 1 and 2 Are Differentially Associated With Auditory Nerve and Spinal Trigeminal Inputs to the Cochlear Nucleus
}

\author{
JIANXUN ZHOU, NAVEEN NANNAPANENI, AND SUSAN SHORE* \\ Kresge Hearing Research Institute, Department of Otolaryngology, University of \\ Michigan, Ann Arbor, Michigan 48109-0506
}

\begin{abstract}
Projections of glutamatergic somatosensory and auditory fibers to the cochlear nucleus (CN) are mostly nonoverlapping: projections from the spinal trigeminal nucleus (Sp5) terminate primarily in the granule cell domains (GCD) of $\mathrm{CN}$, whereas type I auditory nerve fibers (ANFs) project to the magnocellular areas of the VCN (VCNm) and deep layers of Dorsal CN (DCN). Vesicular glutamate transporters (VGLUTs), which selectively package glutamate into synaptic vesicles, have different isoforms associated with distinct subtypes of excitatory glutamatergic neurons. Here we examined the distributions of VGLUT1 and VGLU2 expression in the $\mathrm{CN}$ and their colocalization with Sp5 and ANF terminals following injections of anterograde tracers into Sp5 and the cochlea in the guinea pig. The CN regions that showed the most intense expression of VGLUT1 and VGLUT2 were largely nonoverlapping and were consistent with ANF and Sp5 projections, respectively: VGLUT1 was highly expressed in VCNm and the molecular layer of the DCN, whereas VGLUT2 was expressed predominantly in the GCD. Half $(47 \% \pm 3 \%)$ of the Sp5 mossy fiber endings colabeled with VGLUT2, but few $(2.5 \% \pm 1 \%)$ colabeled with VGLUT1. In contrast, ANFs colabeled predominantly with VGLUT1. The pathway-specific expression of VGLUT isoforms in the CN may be associated with the intrinsic synaptic properties that are unique to each sensory pathway. J. Comp. Neurol. 500:777-787, 2007. @ 2006 Wiley-Liss, Inc.
\end{abstract}

Indexing terms: glutamatergic; multisensory integration; plasticity; auditory; cochlear nucleus; trigeminal

Projections from the trigeminal sensory complex to the cochlear nucleus (CN; Haenggeli et al., 2005; Zhou and Shore, 2004) are integrated with auditory inputs from the cochlea (Shore, 2005). Synaptic terminals of fibers from the spinal trigeminal nuclei (Sp5) and trigeminal ganglion are mostly confined to the granule cell domain (GCD) of the CN. The GCD includes both the superficial shell region of the ventral $\mathrm{CN}$ (VCN) and the fusiform cell layer of dorsal cochlear nucleus (DCN) and contains numerous small cells, including granule cells (Weedman et al., 1996; Zhou and Shore, 2004). The Sp5 projection gives rise to small boutons and large, irregular swellings (i.e., mossy fibers; MFs) that make contacts with granule cells (Haenggeli et al., 2005; Zhou and Shore, 2004). Together with other somatosensory pathways to the CN, the Sp5 projection conveys proprioceptive information related to head/neck position and vocal tract gestures that are necessary for facilitating sound localization and attenuating body-generated sounds (Kanold and Young, 2001; Shore, 2005; Shore and Zhou, 2006). Somatosensory projections to $\mathrm{CN}$ have also been linked to tinnitus by the observations that tinnitus patients can modulate their tinnitus with head and neck manipulations (Levine, 1999).

Identification of neurotransmitters associated with trigeminal projections to $\mathrm{CN}$ is necessary for a better under-

Grant sponsor: National Institute on Deafness and Other Communication Disorders; Grant number: R01 DC DC004825; Grant number: P30 DC-05188; Grant sponsor: Tinnitus Research Consortium.

*Correspondence to: Susan Shore, Kresge Hearing Research Institute, Department of Otolaryngology, University of Michigan, 1301 East Ann Street, Ann Arbor, MI 48109-0506. E-mail: sushore@umich.edu

Received 4 May 2006; Revised 10 August 2006; Accepted 12 September 2006

DOI 10.1002/cne.21208

Published online in Wiley InterScience (www.interscience.wiley.com). 
standing of multisensory integration in the CN. Earlier studies demonstrated that MFs in the $\mathrm{CN}$ originating in the cuneate nucleus contain round synaptic vesicles, make asymmetric synapses with postsynaptic targets, and are labeled with an antibody to glutamate, suggestive of a glutamatergic pathway (Wright and Ryugo, 1996). MFs from other sources, such as the pontine nucleus, have similar ultrastructural characteristics (Ohlrogge et al., 2001; Weedman et al., 1996). The evidence presented in this paper suggests that the Sp5 pathway to the CN is also glutamatergic.

Vesicular glutamate transporters (VGLUTs) selectively package glutamate into synaptic vesicles and mediate glutamate transport. VGLUTs therefore serve as excellent markers of glutamatergic neurons. Three subtypes, VGLUT1, VGLUT2, and VGLUT3, have been identified in the central nervous system. Although they are structurally homologous and accumulate glutamate with similar transport kinetics (Fremeau et al., 2002; Gras et al., 2002; Takamori et al., 2001), their distributions in the brain are distinct. Both VGLUT1 and VGLUT2 are abundantly expressed in the brain and account for the majority of known glutamatergic neurons in the central nervous system, whereas VGLUT3 is restricted to small, often nonglutamatergic populations of neurons in the brain. Furthermore, VGLUT1 and VGLUT2 show mutually exclusive distributions in the adult mammalian brain: VGLUT1 is intensely expressed in the cerebral and cerebellar cortices and hippocampus, whereas VGLUT2 is expressed predominantly in the thalamus, deep cerebellar nuclei, and many brainstem regions (Fremeau et al., 2001; Herzog et al., 2001; Kaneko et al., 2002; Takamori et al., 2001).

Both VGLUT1 and VGLUT2 (Herzog et al., 2004; Kaneko et al., 2002), but not VGLUT3, are expressed in the $\mathrm{CN}$ in the adult rodent (Herzog et al., 2004). The two VGLUT isoforms show little overlapping expression in the CN: VGLUT1 is more intense in the core region of VCN, whereas VGLUT2 expression is stronger in the shell regions of VCN (Kaneko et al., 2002). Since the GCD is the major recipient of Sp5 and other somatosensory inputs (Haenggeli et al., 2005; Zhou and Shore, 2004), this suggests the association of VGLUT2 with nonauditory inputs to the $\mathrm{CN}$. In contrast, the core region of $\mathrm{CN}$ receives input from glutamatergic type I auditory nerve fibers (ANFs; Altschuler et al., 1984; Brown and Ledwith, 1990; Liberman, 1993; Martin, 1985; Schweitzer et al., 1991). It is thus likely that type I ANFs use VGLUT1 to mediate the glutamate transport. In the present study, our primary goal was to identify whether glutamate is associated with trigeminal projections to CN. Concurrently, we examined the differential distributions of VGLUT2 and VGLUT1

\section{Abbreviations}

DCN

DCN1

DCN2

DCN3

PVCN

SOC

Sp5

Sp5C

Sp5I

$\mathrm{VCN}$

$\mathrm{VCNm}$ dorsal cochlear nucleus

molecular layer of DCN

fusiform cell layer of DCN

deep layer of DCN

posteroventral cochlear nucleus

superior olive complex

spinal trigeminal nucleus

pars caudalis of spinal trigeminal nucleus

pars interpolaris of spinal trigeminal nucleus

ventral cochlear nucleus

magnocellular cell areas of VCN expression in the $\mathrm{CN}$ and their association with the Sp5 and ANF projections to the CN. Differential association of VGLUT isotypes with Sp5 and ANF projections to the CN demonstrated in this study warrant further investigation of other nonauditory afferents to the CN.

\section{MATERIALS AND METHODS Animal preparation}

The experiments were performed on 12 pigmented guinea pigs, weighing 300-400 g. All procedures were performed in accordance with the NIH guidelines for the use and care of laboratory animals (NIH publication No. 80-23) and guidelines provided by the University Committee on Use and Care of Animals (UCUCA) of University of Michigan.

Four animals were used for studying VGLUT immunoreactivity (VGLUT-ir) in the $\mathrm{CN}$. The animals were euthanized with Nembutal (15 mg/kg, i.p.) and transcardially perfused with $0.1 \mathrm{M}$ phosphate-buffered saline (PBS), followed by $4 \%$ paraformaldehyde in PBS. After perfusionfixation, the brain was isolated and placed in the same fixative for 2 hours at $4^{\circ} \mathrm{C}$. The brain was transferred into $30 \%$ sucrose in $0.1 \mathrm{M}$ PBS overnight at $4^{\circ} \mathrm{C}$, and the brainstem was then sectioned on a freezing microtome at a thickness of $40 \mu \mathrm{m}$.

Eight guinea pigs were used in a double-labeling fluorescence study to identify the colocalization of VGLUT1-ir and VGLUT2-ir with the terminals of Sp5 fibers $(n=6)$ and ANFs $(n=2)$. These animals were anesthetized with intramuscular injections of ketamine hydrochloride (Ketaset; $40 \mathrm{mg} / \mathrm{kg}$ ) and xylazine (Rompun; $10 \mathrm{mg} / \mathrm{kg}$ ) and placed in a stereotaxic frame (David Kopf, Tujunga, CA). Rectal temperature was maintained at $38^{\circ} \mathrm{C} \pm 0.5^{\circ} \mathrm{C}$ with a thermostatically controlled heating pad. The surgical procedure for injections of anterograde tracers in the Sp5 has been described previously (Zhou and Shore, 2004). A total volume of $0.1-0.3 \mu \mathrm{l}$ tracer $[10 \%$ biotinylated dextran-amine (BDA); MW 10,000: Molecular Probes, Eugene, OR; or Fluoro-Ruby (FR): Molecular Probes] was sterotaxically pressure-injected into the Sp5 with a Hamilton microsyringe equipped with a glass micropipette $(20-30 \mu \mathrm{m}$ tip). Tracer injections into the cochlea [Fluoro-Gold (FG): Fluorochrome, LLC, Denver, CO] were achieved by making a small hole in the round window membrane, which was then plugged with a small, tracer-soaked piece of gelatin sponge (Shore and Moore, 1998). Four to six days after the injections, animals were killed, and the brains were processed and sectioned as described above.

\section{Tissue processing and immunocytochemistry}

Sections were mounted in serial order on clean glass slides and air dried. Alternate serial sections were used to label VGLUT1 and VGLUT2. All tissue processing was at room temperature $\left(20-22^{\circ} \mathrm{C}\right)$ unless otherwise stated. Polyclonal antibodies were generated in rabbits against Strep-Tag fusion protein containing amino acid residues 456-560 of rat VGLUT1 (Synaptic Systems; catalog No. 135 303) and Strep-Tag fusion protein containing amino acid residues 510-582 of rat VGLUT2 (Synaptic Systems; catalog No. 135 403). Both primary antibodies and secondary antibody (Alexa Fluor 488-conjugated goat anti-rabbit; Molecular Probes) were diluted in the same blocking solution containing $1 \%$ normal goat serum (Jackson Im- 

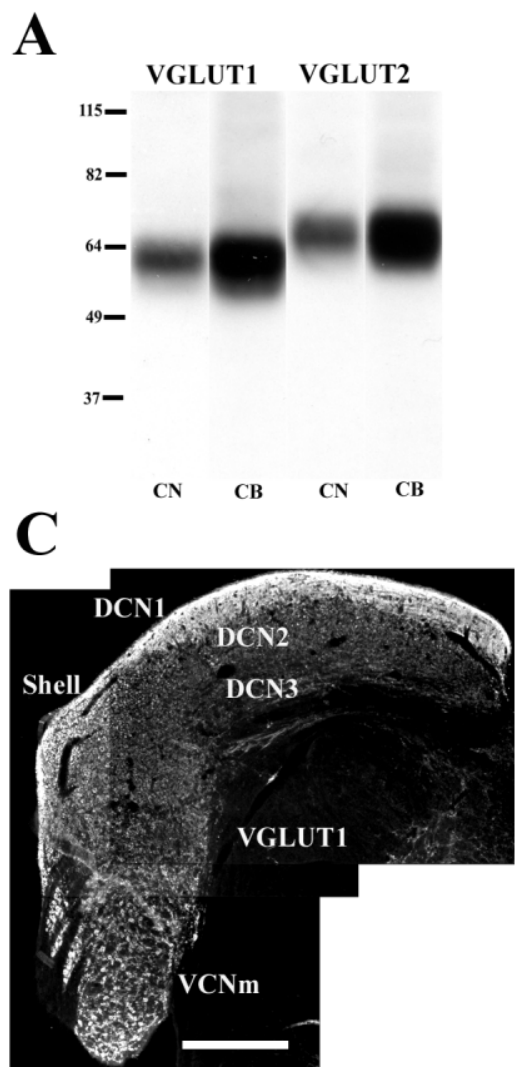

Fig. 1. Expression of VGLUT1 and VGLUT2 in the CN. A: Western blot analysis of proteins from $\mathrm{CN}$ and cerebellum (CB) with anti-VGLUT1 and anti-VGLUT2 antibodies. Anti-VGLUT1 antibody recognized a single band at $\sim 60 \mathrm{kDa}$, and anti-VGLUT2 antibody recognized a single band at $\sim 65 \mathrm{kDa}$, corresponding to the molecular weights predicted for VGLUT1 and VGLUT2, respectively. Molecular weight standards are indicated at left $(\mathrm{kDa})$. B: VGLUT1-ir and VGLUT2-ir in the cerebellar cortex, as the positive control (M, molec-
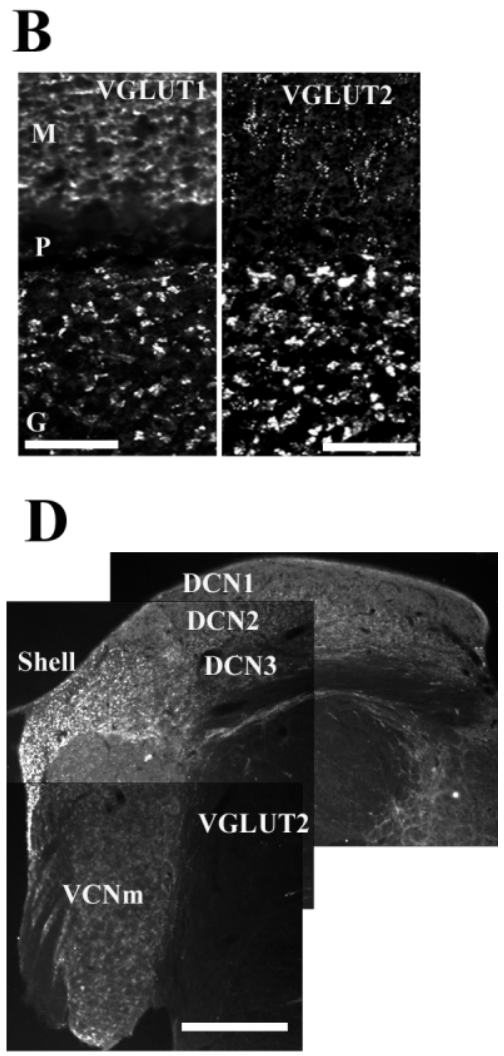

ular layer; P, Purkinje cell layer; G, granular layer). C: VGLUT1-ir in the $\mathrm{CN}$ at low magnification $(\times 10)$. VGLUT1 is intensely expressed in DCN1 and VCNm; weak to moderate VGLUT1-ir is found in the shell and DCN2; weak VGLUT1-ir is seen in DCN3. D: VGLUT2-ir in the $\mathrm{CN}$ at low magnification $(\times 10)$. VGLUT2 is expressed predominantly in the shell; moderate VGLUT2-ir is found in DCN2; very weak to weak staining is found in DCN1, VCNm, and DCN3. Scale bars $=50$ $\mu \mathrm{m}$ in $\mathrm{B} ; 0.5 \mathrm{~mm}$ in $\mathrm{C}, \mathrm{D}$. munoresearch, West Grove, PA; 005-000-121) in 0.1 M PBS with 0.1\% Triton X-100 (Sigma, St. Louis, MO; 900293-1), pH 7.4. The sections were incubated in the blocking solution for 30 minutes, followed by overnight incubation with primary antibodies (1:1,000 for both VGLUT1 and VGLUT2). After thoroughly rinsing in PBS, sections were reacted for 2 hours with the secondary antibody. Finally, sections were rinsed, dehydrated in graded ethanol, and coverslipped with Micro-cover (Micron, Fairfax, VA). Negative controls were conducted on sections that were not treated with either primary or secondary antibodies, resulting in no immunolabeling. Preincubation of VGLUT antibodies with corresponding synthetic peptides (Strep-Tag fusion protein containing amino acid residues 456-560 of rat VGLUT1, catalog No. 135-3P; and Strep-Tag fusion protein containing amino acid residues 510-582 of rat VGLUT2, catalog No. 135-4P; Synaptic Systems) resulted in negative immunolabeling. VGLUT expression in the cerebellar cortex had been well documented (Hioki et al., 2003; Kaneko et al., 2002; Takamori et al., 2001), so cerebellar cortex was used as the positive control. To visualize BDA-labeled Sp5 terminals colabeled with VGLUT-ir, sections were incubated for 2 hours with rhodamine red-X conjugated with streptavidin (S6366; Molecular Probes), followed by immunolabeling with VGLUTs.

\section{Western blots}

Brain tissue (cerebellum and $\mathrm{CN}$ ) was collected and homogenized with a sonicator in an ice-cold lysis buffer (1\% NP40 in PBS) with protease inhibitors (Roche Molecular Biochemicals, Indianapolis, IN). The homogenate was centrifuged for 5 minutes at 14,000 rpm, and total protein concentration was measured with the Bio-Rad Protein Assay (Bio-Rad, Hercules, CA). Equal concentrations of protein $(25 \mu \mathrm{g}$ per lane) were separated by SDS-PAGE and transferred to a nitrocellulose membrane. After blocking with PBS-Tween 20 containing 5\% nonfat dry milk, immunoblotting with primary antibodies was performed (1: 3,000 for both anti-VGLUT1 and anti-VGLUT2); secondary antibodies (anti-rabbit-HRP from Sigma) were diluted at 1:10,000 in PBS-Tween 20 with $5 \%$ milk. Signals were then revealed with ECL (Amersham Pharmacia Biotech, Arlington Heights, IL) and exposed to Hyperfilm (Amersham Pharmacia Biotech). 

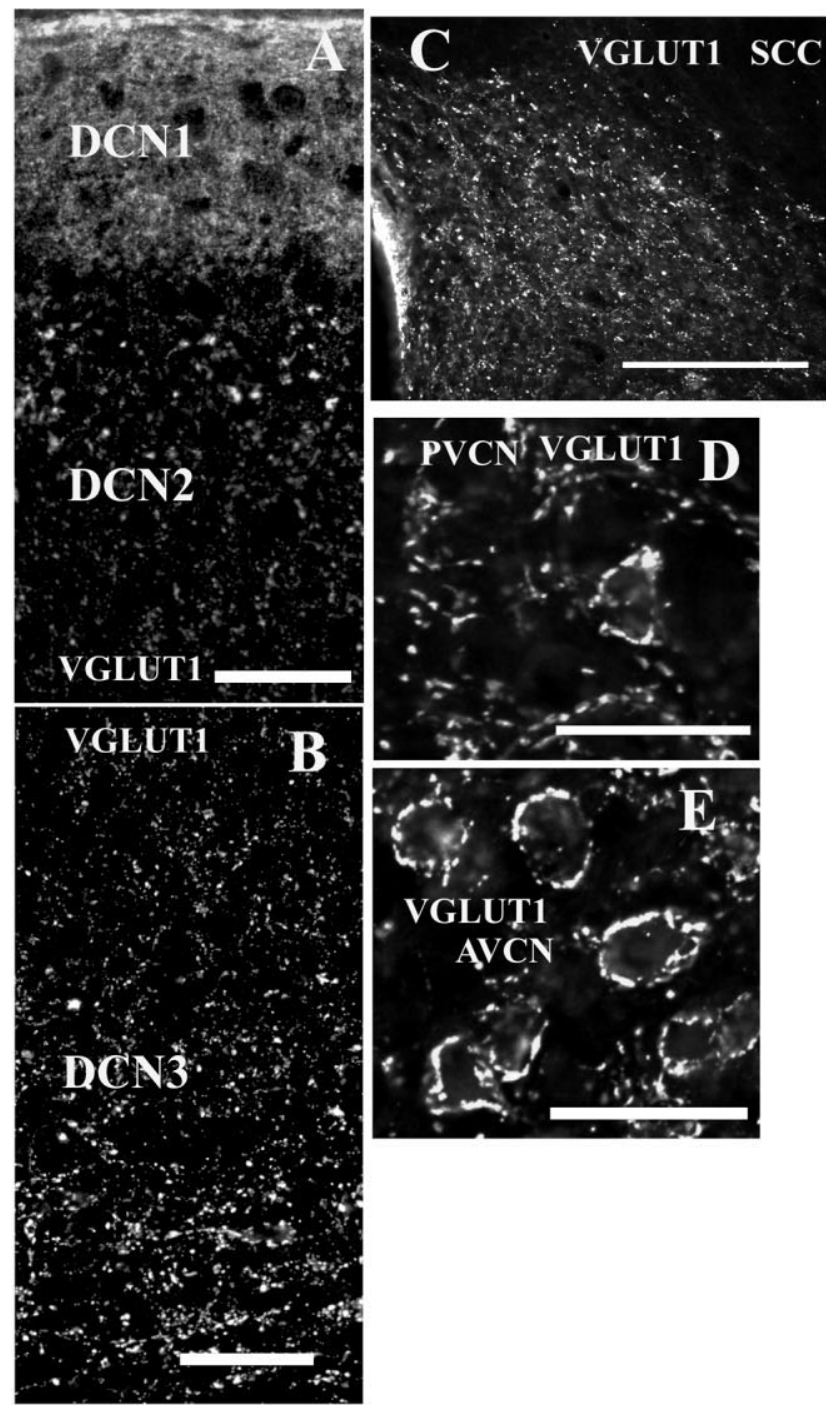

Fig. 2. Photomicrographs of VGLUT1-ir and VGLUT2-ir in the CN at higher magnifications $(\times 20$ : A-C,F-H; $\times 40$ : D,E,I,J). A-E: Different forms of VGLUT1 labeling in CN subdivisions: small, densely distributed granules in DCN1 (A); small to medium-sized granules in DCN3 (B) and other CN subdivisions $(\mathrm{C}-\mathrm{E})$; large irregular granules in DCN2 (A) and shell (C); endbulb-like structures around large somata in the VCNm (D,E). F-J: Different forms of VGLUT2 labeling

\section{Image processing and confocal laser microscopy}

Sections were examined with a fluorescent microscope equipped with the appropriate filters for rhodamine and Alexa fluor 488 (Leica, DM). Photomicrographs of immunolabeling were digitized and imported to Metamorph for quantification of immunolabeling. The parameters for digitizing photomicrographs were determined in a pilot study with optimal contrast between labeling and background and were then kept constant across conditions and animals. Photomicrographs were imported into Adobe Photoshop for contrast adjustment.

Analysis of VGLUT-ir was conducted for all subdivisions of the $\mathrm{CN}$, with emphasis on the following regions:
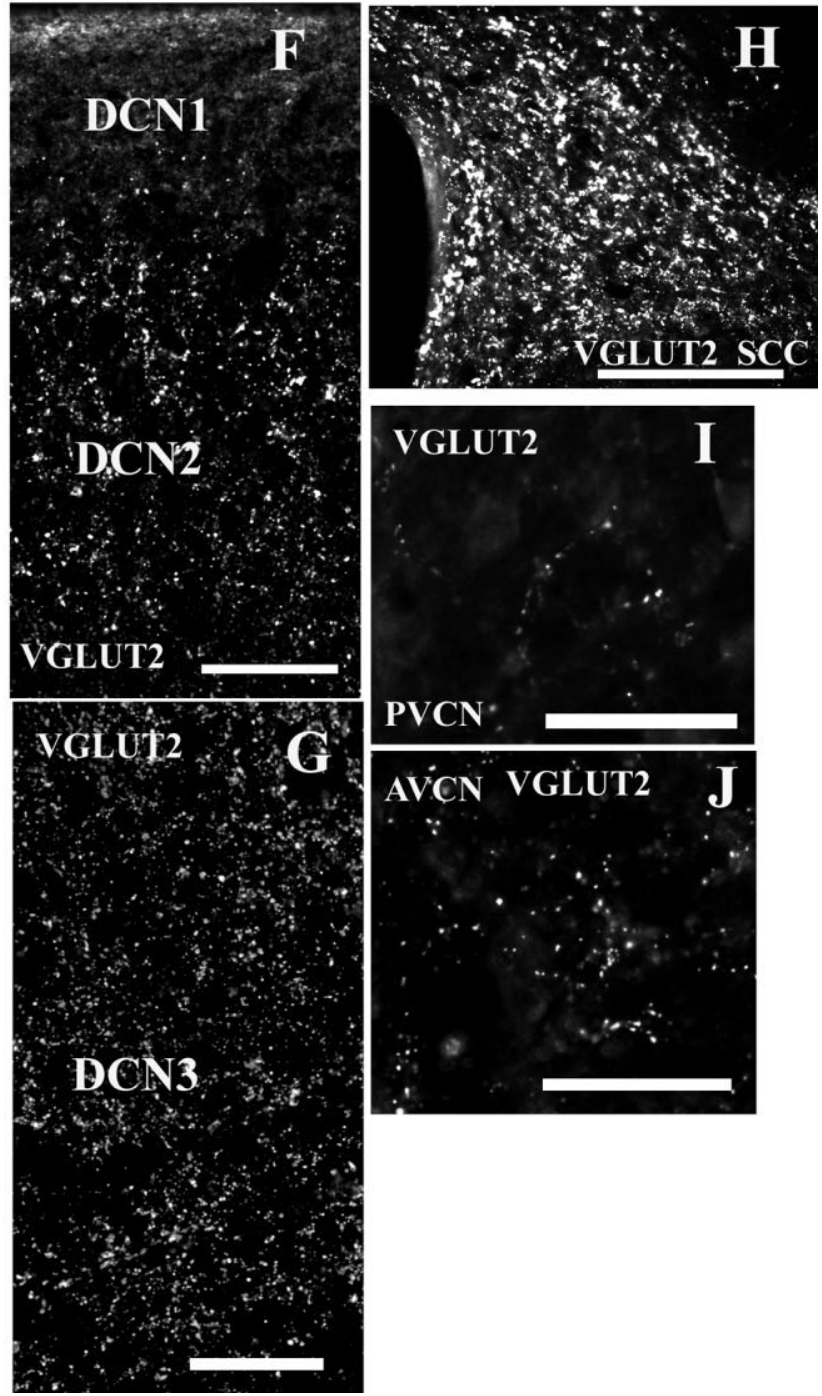

in CN subdivisions: large, irregular granules in the DCN2 (F) and shell $(\mathrm{H})$; small to medium-sized granules in DCN3 (G) and VCNm $(\mathrm{I}, \mathrm{J})$. The large irregular VGLUT2-labeled granules in the shell appeared larger and more intense than the VGLUT1-labeled granules in the same $\mathrm{CN}$ region $(\mathrm{H})$. Scale bars $=50 \mu \mathrm{m}$ in A,B,D-G,I,J; $100 \mu \mathrm{m}$ in $\mathrm{C}, \mathrm{H}$.

the DCN molecular layer (DCN1), the fusiform cell layer (DCN2), the deep DCN (DCN3), the VCN magnocellular areas $(\mathrm{VCNm})$, and the shell region of $\mathrm{VCN}$. In the doublelabeling study, alternate serial sections were used to label VGLUT1 and VGLUT2, respectively. Under epifluorescence, the labeled Sp5 terminals were manually counted on alternate sections in each group. Double labeling of Sp5 terminals with VGLUT-ir was determined by frequently switching the filters and adjusting the focusing of the objective lens. Colocalization was established when the two different labels (VGLUT and Sp5) exactly marked the same profile at the same focusing level. The number of terminal counts was multiplied by 4 to achieve the total terminal counts and corrected for double-counting errors 


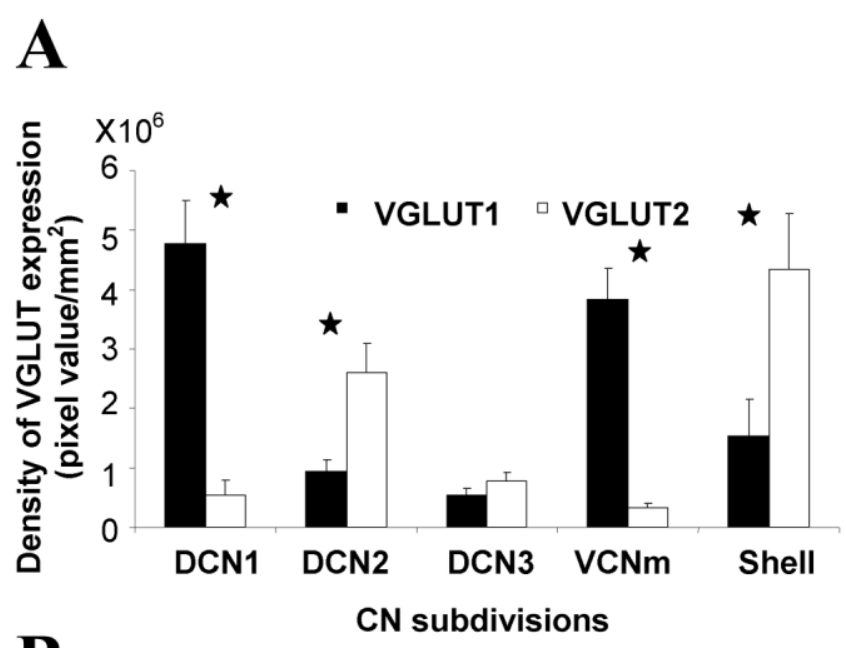

B

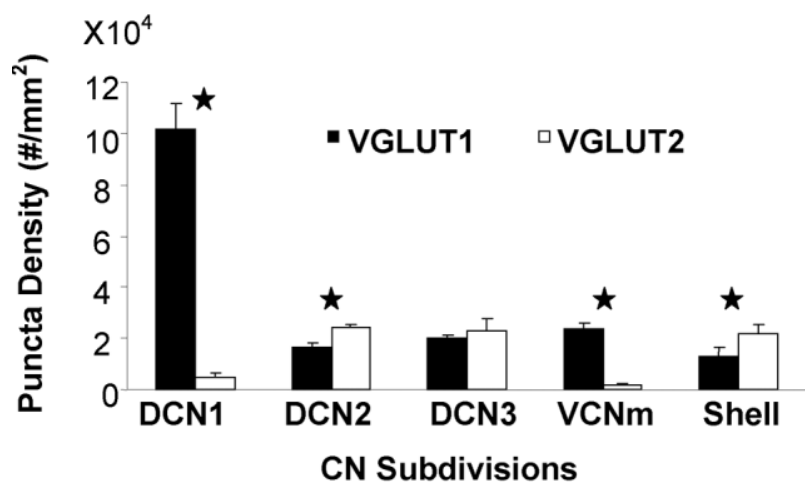

Fig. 3. Quantification of the VGLUT1-ir and VGLUT2-ir in the CN $(\mathrm{n}=4)$. A: Density of VGLUT expression in the CN. B: Puncta density of the VGLUT1-ir and VGLUT2-ir in the CN. Error bars represent SEM. Asterisks indicate significant differences (see text).

by using Abercrombie's correction: corrected number = count $\times$ [section thickness/(section thickness + terminal size)] (Abercrombie, 1946). The labeled Sp5 terminal endings were classified as: 1) MF-like terminal endings: large irregular swellings ( $\geq 2 \mu \mathrm{m})$, which usually give rise to collaterals; 2$)$ small boutons: small and round or oval $(<2$ $\mu \mathrm{m}$ ), including en passant and terminal boutons (Zhou and Shore, 2004).

In some cases, double-labeled immunofluorescent terminals were also identified by confocal laser microscopy (Zeiss LSM 510). The images immunolabeled with Alexa 488 and rhodamine were scanned with lasers of argon and $\mathrm{HeNe} 1$, respectively. The images immunolableled with FG were scanned with a UV laser with an excitation wavelength of $364 \mathrm{~nm}$, filtered using an emission LP filter at $560 \mathrm{~nm}$, and viewed on the red channel. Images were acquired at a $1-\mu \mathrm{m}$ optical thickness. To obtain a clear view of terminal profiles, a series of six to 11 serial optical sections was obtained to generate a Z-projection of stacks. The double-labeled ANF terminals were counted manually from photomicrographs of confocal images $(\times 63)$ of $1-\mu \mathrm{m}$ optical thickness. Two sections were selected from both the DCN3 and the VCNm. The labeled ANF terminal endings were classified as 1) endbulb-like terminals, a group of puncta aligned in a circle or semicircle around a large cell body, or 2) bead-like terminals, small and round or oval $(<2 \mu \mathrm{m})$, including en passant and terminal boutons. Puncta that were yellow on the confocal multichannel images indicated colocalization.

\section{Quantification and statistical analysis}

To quantify VGLUT-ir in the CN, three sections were selected from each of the following regions: the DCN, magnocellular VCN, and shell region. For each of these regions, sections spanned evenly from rostral to caudal (i.e., one section from the 25 th percentile, one from the median, and one from the 75 th percentile). For VCN and shell region, two pictures $(\times 40)$ were taken of each selected section. For DCN, layers 1, 2, and 3 were photographed separately, yielding a total of six photomicrographs $(\times 40)$ for each chosen DCN section. In addition, two pictures with higher magnification $(\times 63)$ were taken of DCN1 at each selected level for the purpose of manual counting (see below). Quantification for each region (DCN, $\mathrm{VCN}$, and shell region) was based on three sections/ regions in each animal. The photomicrographs were then transferred to Metamorph for quantification. The number of puncta and the pixel intensity of VGLUT-ir were semiautomatically measured: To ensure that puncta were counted reliably, visual inspections and manual corrections were always conducted after each automated counting. Because of the high density of VGLUT1-ir in DCN1, VGLUT-labeled puncta (VGLUT1 and 2) were counted manually in a chosen area by using photomicrographs with higher magnification $(\times 63)$. The number of VGLUTlabeled puncta was divided by the chosen area to yield the puncta density.

For quantitative assessment of intensity of VGLUT expression, the overall pixel value of VGLUT-ir for each photomicrograph was first corrected by subtracting the overall pixel value for a negative control section (i.e., without primary antibodies). The corrected value was then divided by the chosen area of counting, yielding the intensity per unit area (i.e., density of VGLUT expression expressed as pixel value $/ \mathrm{mm}^{2}$ ). Pixel densities less than $1 \times$ $10^{6}$ pixel value $/ \mathrm{mm}^{2}$ were defined as very weak, $1-2 \times 10^{6}$ pixel value $/ \mathrm{mm}^{2}$ as weak, $2-4 \times 10^{6}$ pixel value $/ \mathrm{mm}^{2}$ as moderate, and $>4 \times 10^{6}$ pixel value $/ \mathrm{mm}^{2}$ as intense.

Means and SEMs were calculated for the following: 1) puncta density of VGLUT1- and VGLUT2-ir, 2) density of VGLUT expression, 3) number of Sp5-labeled terminals, 4) number of Sp5-labeled terminals colabeled with VGLUT1 and VGLUT2, and 5) percentage ratios of Sp5 terminals colabeled with VGLUT1 and VGLUT2. Student's paired $t$-test was used to perform comparisons between means.

\section{RESULTS \\ VGLUT expression in the CN}

Western blot analysis with anti-VGLUT1 antibody showed a single band at $\sim 60 \mathrm{kDa}$ on protein extracted from both $\mathrm{CN}$ and cerebellum (CB; Fig. 1A), and antiVGLUT2 antibody recognized a single band at $\sim 65 \mathrm{kDa}$, corresponding to the molecular weights predicted for VGLUT1 and VGLUT2, respectively. Positive controls for VGLUT-ir in the cerebellar cortex revealed dense, granular VGLUT1-ir and sparse, beaded VGLUT2-ir in the molecular layer (M in Fig. 1B), and coarse VGLUT1 and 


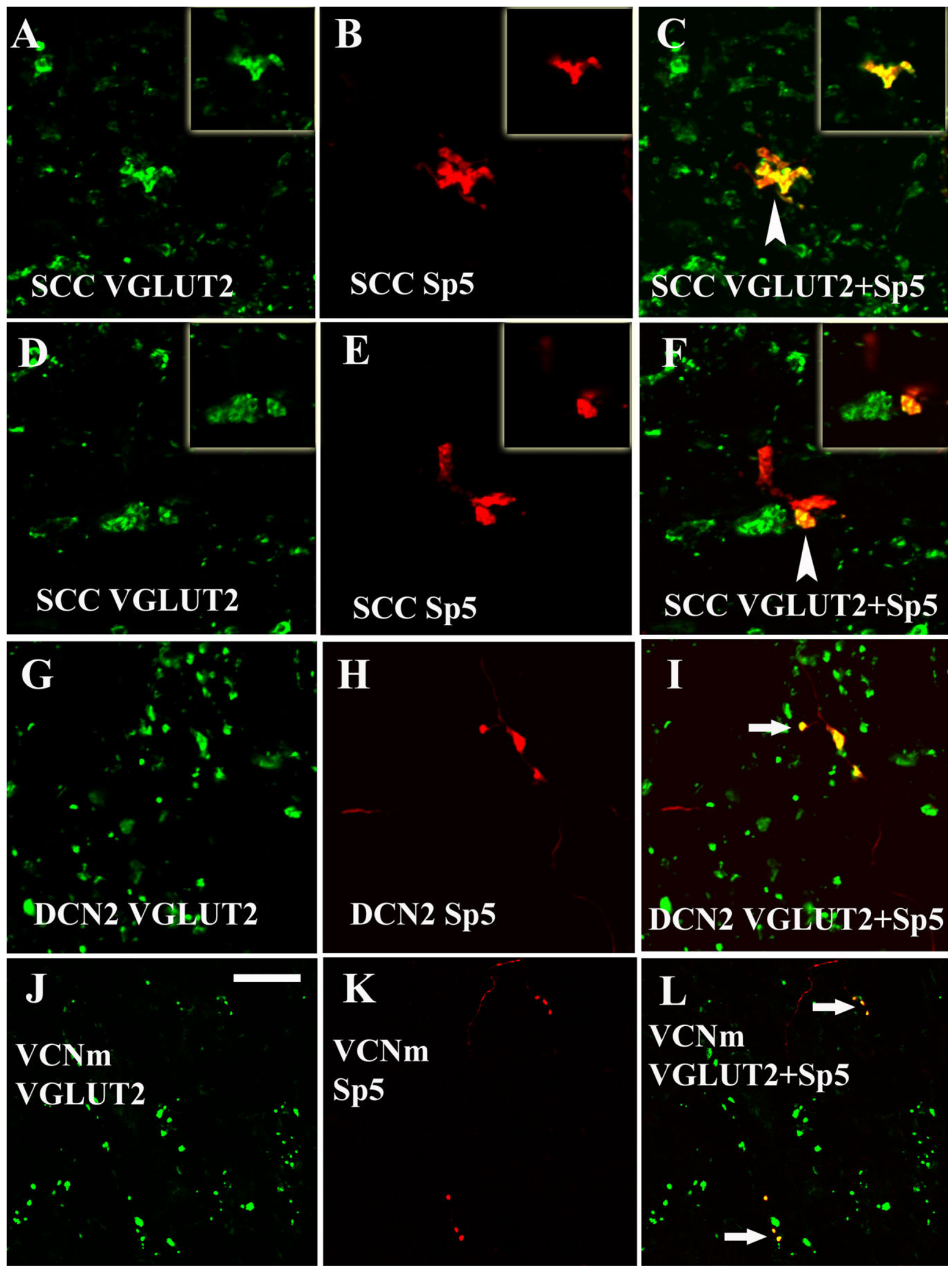


VGLUT2-ir in the granular layers (G in Fig. 1B), as previously demonstrated (Kaneko et al., 2002; Nunzi et al., 2003). The most intense VGLUT1-ir was in the molecular layer of DCN (Fig. 1C, DCN1) and magnocellular area of VCN (VCNm, Fig. 1C). Weak to moderate VGLUT1-ir was observed in the shell region of VCN and DCN2. VGLUT1-ir in DCN3 was weak or very weak. In contrast, the most intense VGLUT2-ir was in the shell region (Fig. 1C). VGLUT2-ir was moderate in DCN2 and weak or very weak in DCN1, DCN3, and VCNm (Fig. 1D).

VGLUT1 labeling was in the form of small granules, densely distributed in DCN1 (Fig. 2A) and also scattered in other CN subdivisions (Fig. 2A-E). Large, irregular granules in DCN2 and shell region (Fig. 2C) or endbulblike structures around large somata in VCNm were also observed (Fig. 2D,E). The small granules in DCN1 and the endbulb-like structures around large cell bodies in VCNm were the major types of VGLUT1-labeled endings in the CN. VGLUT2 labeling, on the other hand, was mainly in the form of large, irregular granules in DCN2 and shell region (Fig. $2 \mathrm{~F}-\mathrm{J}$ ) that were larger and more intense than VGLUT1-labeled granules in the same CN region (Fig. $2 \mathrm{H})$. Small to medium-sized VGLUT2-labeled granules were observed in the DCN2 and DCN3 (Fig. 2F,G), shell region (Fig. 2H), and VCNm (Fig. 2I,J).

\section{Quantification of VGLUT-ir in CN}

VGLUT2-ir was significantly more intense than VGLUT1-ir in the shell region and DCN2 (Fig. 3A; paired $t$-test, $P<0.01$ ), whereas VGLUT1-ir was significantly more intense than VGLUT2-ir in the VCNm and DCN1 (Fig. 3A; paired $t$-test, $P<0.001$ ). Similarly, the puncta density of VGLUT2-labeled granules was significantly greater than that of VGLUT1-labeled granules in the shell region and DCN2 (Fig. 3B; paired $t$-test, $P<0.05$ ), whereas the puncta density of VGLUT1-labeled granules was significantly greater than that of VGLUT2-labeled granules in the VCNm and DCN1 (Fig. 3B; paired $t$-test, $P<0.01$ ).

\section{Sp5 terminals colabel with VGLUT2}

Six animals were injected with anterograde tracers in the ipsilateral Sp5 (Sp5I, pars interpolaris). Data analysis focused on two animals in which the injections were limited to Sp5I, without diffusion to adjacent areas. In the other four animals, some spread to the adjacent inferior cerebellar peduncle (icp) and the lateral medullar reticular formation occurred. We have previously demonstrated that the icp does not contribute to the Sp5-CN projection (Zhou and Shore, 2004). Although the medullar reticular

\footnotetext{
Fig. 4. High-magnification confocal images $(\times 63)$ showing colocal ization of anterogradely labeled Sp5 terminal endings with VGLUT2-ir in different regions of the CN. Green, VGLUT-ir; red, Sp5 labeling; yellow, double-labeled terminals. A-F were obtained from Z projections of stacks of serial $1-\mu \mathrm{m}$ confocal images and $\mathbf{G}-\mathbf{L}$ each show a single, $1-\mu \mathrm{m}$ confocal image. Insets in A-F show a single 1- $\mu \mathrm{m}$ confocal image. A-F: MFs are labeled with BDA from Sp5 and VGLUT2 in the shell. Colocalization of Sp5 MFs with VGLUT2-ir is indicated by arrowheads in C,F. G-I: Small boutons are labeled with BDA from Sp5 and VGLUT2 in DCN2. Colocalization of Sp5 small boutons with VGLUT2 is shown by arrows in I. J-L: Small boutons are labeled with BDA from Sp5 and VGLUT2 in VCNm. Colocalization of Sp5 small boutons with VGLUT2-ir is shown by arrows in L. Scale bar $=10 \mu \mathrm{m}$
}

formation does project to the CN (Shore and Zhou, 2006), the region that contains the most $\mathrm{CN}$ projection neurons is rostral to the Sp5I and Sp5C (pars caudalis). In the present study, all injections were located in the intermediate to caudal part of Sp5I. In addition, the distribution, morphology, and colocalization of the labeled terminal endings with VGLUT-ir from these four animals were similar to those from animals with restricted injections. Therefore, contributions from lateral medulla reticular formation to the terminal labeling in the $\mathrm{CN}$ were minimal. Nonetheless, the quantification analysis was based only on the two animals in which the injections were restricted to the Sp5.

Colocalization of Sp5 endings with VGLUT2. The most prominent Sp5 labeled terminals were large, irregular swellings ( $\geq 2 \mu \mathrm{m}$ ), characteristic of MFs (Weedman et al., 1996; Wright and Ryugo, 1996), in the GCD (Figs. 4A-F, 6A). Sp5 MFs that colabeled with VGLUT2 (47.7\% $\pm 3 \%$, Figs. 4, 6C) were restricted to the GCD (Fig. 6B), which receives the majority of Sp5 terminals (Zhou and Shore, 2004). These endings did not show morphological or topographical differences from those that did not colabel with VGLUT2 (Fig. 4D-F). A lower percentage of the small Sp5 boutons $(<2 \mu \mathrm{m})$ located throughout the CN colabeled with VGLUT2 $(11.5 \% \pm 0.5 \%$ in the GCD, and $12.1 \% \pm 8 \%$ in the VCN; Figs. 4G-L, 6B). No colocalization of Sp5 boutons with VGLUT2-ir was evident in DCN1 and DCN3 (Fig. 6B).

Lack of colocalization of Sp5 endings with VGLUT1. In strong contrast, the majority of labeled Sp5 terminals, including MFs and small boutons, did not colocalize with VGLUT1-ir (Figs. 5, 6). Few Sp5 MFs $(2.5 \% \pm 1 \%)$ colabeled with VGLUT1, significantly fewer than were colabeled with VGLUT2 $(47.7 \% \pm 3 \%$; paired $t$-test, $P<0.05)$. These VGLUT1-labeled Sp5 MFs did not show morphological or topographical differences from those that did not colabel with VGLUT1 or VGLUT2. No small Sp5 boutons colabeled with VGLUT1. The paucity of colocalization of Sp5 terminals with VGLUT1-ir was evident in all CN subdivisions, even in the DCN1 that showed intense VGLUT1 expression (Fig. 5C). Only $2 \% \pm 0.1 \%$ of total Sp5 terminal endings (both MFs and small boutons) colabeled with VGLUT1, significantly fewer than colabeled with VGLUT2 $(23.6 \% \pm 1 \%$; paired $t$-test, $P<0.05)$.

\section{ANF terminals colabel with VGLUT1}

Infusion of $\mathrm{FG}$ into the labyrinth resulted in heavy labeling of ANFs and their terminals in the VCNm and the DCN3, but only a few labeled axons entered the shell region. The most prominent $\mathrm{ANF}$ terminal endings in the $\mathrm{CN}$ were the endbulb-like endings in the VCNm (Fig. 7). In addition, large numbers of bead-like terminal boutons were evident in the VCNm and the DCN3. Most labeled ANF endbulb-like terminals (Fig. 6D; 79.5\% $\pm 7 \%$ ) colocalized with VGLUT1 in VCNm (Fig. 7A, arrows). Many bead-like ANF terminals or small boutons were also labeled with VGLUT1 in VCNm (Fig. 7A, arrowheads; $43.0 \% \pm 3 \%$; see also Fig. $6 \mathrm{D}$ ) and DCN3 (Fig. 7B, arrowheads; $26.0 \% \pm 5 \%$; see also Fig. $6 \mathrm{D}$ ). These small ANF boutons that were colabeled with VGLUT1 were not morphologically or topographically different from those that were not colabeled with VGLUT1. In contrast, neither ANF endbulb-like terminals (0\%; Fig. 6D) nor small boutons (0\%; Fig. 6D) was colocalized with VGLUT2 (Fig. $7 \mathrm{C}, \mathrm{D})$. 

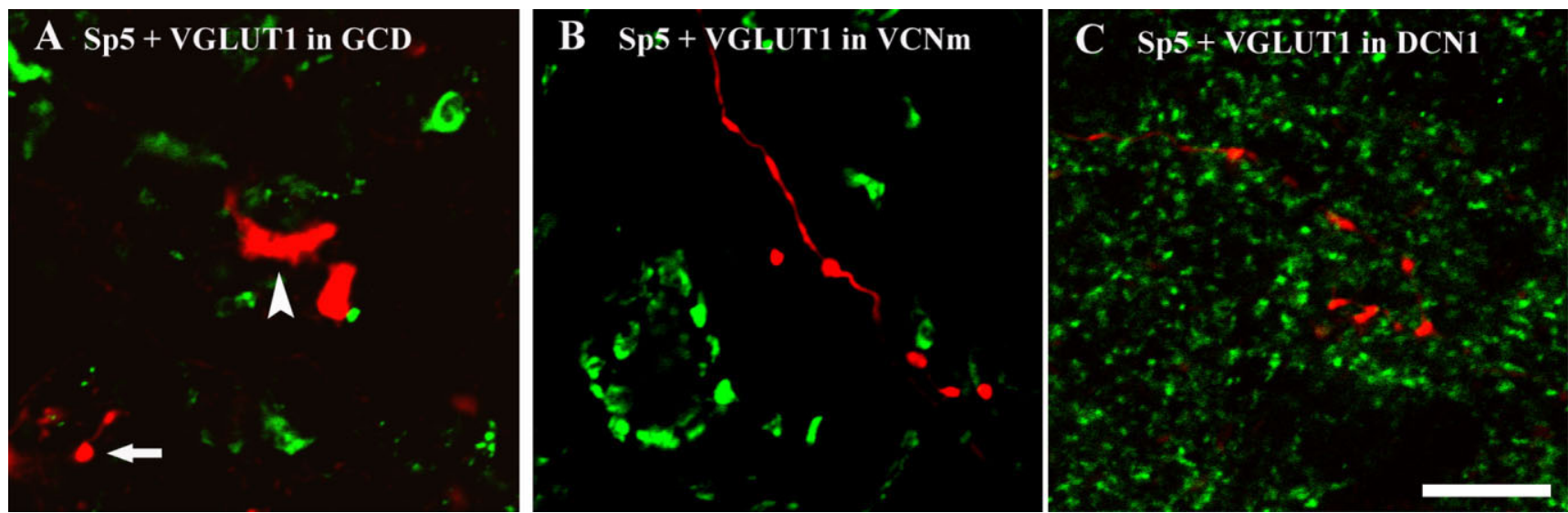

Fig. 5. Sp5 terminal endings do not colabel with VGLUT1. Highmagnification confocal images $(\times 63)$ of Sp5 terminal endings and VGLUT1-ir in different regions of the CN. Green, VGLUT-ir; red, Sp5 labeling; yellow, double-labeled terminals. All figures show a single,
1- $\mu \mathrm{m}$ confocal image. A: Sp5 MFs (arrowhead) and small boutons (arrow) did not colabel with VGLUT1 in the shell. B: Sp5 small boutons did not colabel with VGLUT1 in VCNm. C: Sp5 small boutons did not colabel with VGLUT1 in DCN1. Scale bar $=10 \mu \mathrm{m}$.

\section{DISCUSSION}

This work confirms previous findings that VGLUT expression in the $\mathrm{CN}$ is differentially distributed according to region (Herzog et al., 2001; Kaneko et al., 2002). We extend these findings by demonstrating that the differential distributions of VGLUT1 and VGLUT2 are associated with different sources of afferent inputs to the CN: Sp5 projections were colocalized with VGLUT2, whereas VIIIth nerve projections to the CN were colocalized with VGLUT1.

\section{VGLUT expression in the $\mathrm{CN}$ is associated with different sources of afferent input}

The most intense VGLUT1-ir was seen in the VCNm and DCN1, whereas the most intense expression of VGLUT2 was in the GCD. These CN subdivisions are associated with different sources of afferent inputs: The GCD, encompassing the shell region and DCN2, receives projections from variety of nonauditory sources, including the Sp5, the trigeminal ganglion, reticular formation and the cuneate nucleus (Haenggeli et al., 2005; Li and $\mathrm{Mi}$ zuno, 1997; Ohlrogge et al., 2001; Shore et al., 2000; Weedman et al., 1996; Wright and Ryugo, 1996; Zhou and Shore, 2004; Shore and Zhou, 2006). The magnocellular regions of the $\mathrm{CN}$, including both $\mathrm{VCNm}$ and $\mathrm{DCN} 3$, receive projections from type I ANFs (Brown and Ledwith, 1990; Fekete et al., 1984; Liberman, 1993; Shore and Moore, 1998). The DCN1, the most superficial layer of DCN, comprises predominantly parallel fiber inputs from granule cells in the GCD. Thus, VGLUT1 and -2 may be specifically associated with the synaptic transmission of these different circuits.

The morphological appearances of VGLUT-ir puncta also suggest circuit-specific associations. VGLUT2-ir puncta in the GCD were mostly in the form of large, irregular swellings that resemble MFs. The sources of MF endings include several nonauditory structures, including somatosensory systems and the reticular formation (Haenggeli et al., 2005; Ohlrogge et al., 2001; Shore and Zhou, 2006; Weedman et al., 1996; Wright and Ryugo, 1996; Zhou and Shore, 2004). As shown here, the Sp5 terminal endings in the $\mathrm{CN}$ are either small boutons or large Mfs, which is consistent with previous studies (Haenggeli et al., 2005; Ohlrogge et al., 2001; Wright and Ryugo, 1996; Zhou and Shore, 2004).

In contrast, numerous endbulb-like VGLUT1 labeled terminals from type I ANFs were seen around large cell bodies in the VCNm, suggesting that the ANF glutamatergic projection to $\mathrm{CN}$ is mediated by VGLUT1. The intense, small granular labeling of VGLUT1 in the DCN1 indicates that VGLUT1 is associated with intrinsic connections within the $\mathrm{CN}$, i.e., the parallel fiber-to-DCN projection. This is consistent with the findings that parallel fiber terminals in the cerebellar cortex use VGLUT1 for accumulation of glutamate into the synaptic vesicles (Fremeau et al., 2004a; Kaneko et al., 2002).

\section{VGLUT2 mediates glutamate transport at Sp5 terminals}

The colocalization of VGLUT2-ir with Sp5 terminals demonstrated here indicates that 1) the Sp5 pathway to the $\mathrm{CN}$ is glutamatergic and 2) Sp5 projections to $\mathrm{CN}$ use VGLUT2 to mediate glutamate transport at both their MF- and small bouton terminal endings. MF terminals in the $\mathrm{CN}$ resemble those in the cerebellar cortex. They are located predominantly in the GCD and make synaptic connections with the dendric claws of granule cells (Weedman et al., 1996). Sp5 small boutons, which are more numerous than Sp5 MFs, probably make contacts with cells other than granule cells (Haenggeli et al., 2005; Zhou and Shore, 2004), as observed for bouton terminals from other nonauditory projections to the $\mathrm{CN}$, including those from the cuneate and pontine nuclei (Ohlrogge et al., 2001; Wright and Ryugo, 1996). MFs give rise to collaterals (Haenggeli et al., 2005; Wright and Ryugo, 1996; Zhou and Shore, 2004), so it is likely that small bouton terminals arise not only directly from the Sp5 but also as collaterals from MFs.

Sp5 projections to CN convey somatosensory information pertaining to head and neck position and vocal tract gestures to the CN and can modify the output of DCN projection neurons via the MF-granule cell-fusiform cell circuit. This circuit provides information relating to head 


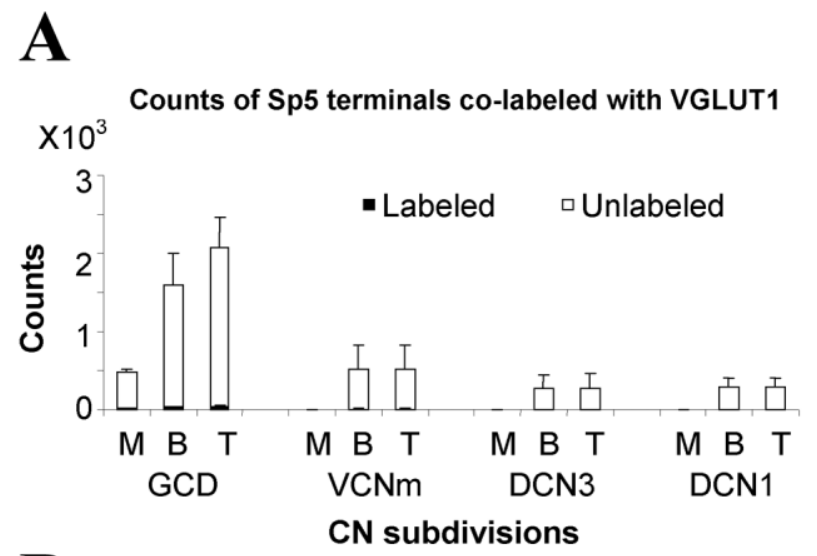

B

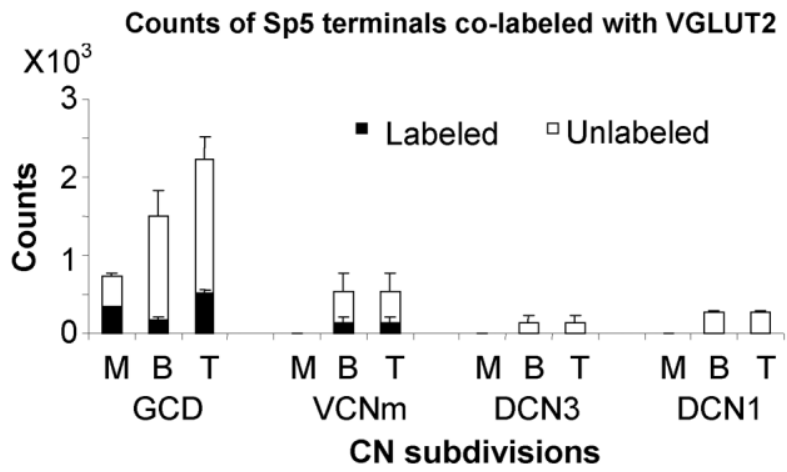

C

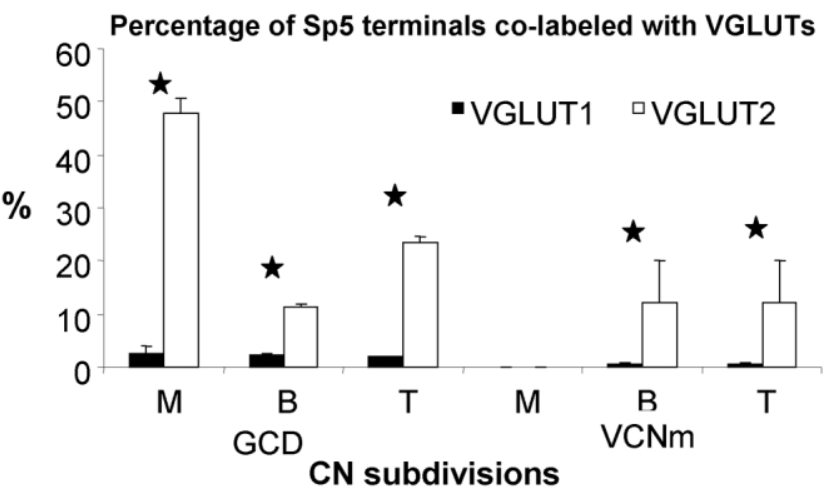

D

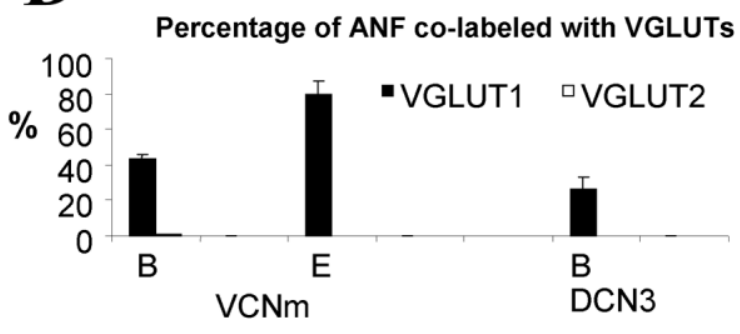

CN subdivisions or neck position to facilitate sound localization (Davis et al., 1996; Kanold and Young, 2001; Young et al., 1995). It may also act as an "adaptive filter" to cancel out internal noise (such as chewing, self-vocalization, and respiration), as suggested by the observation that stimulation of the trigeminal nerve can suppress the DCN responses elicited by sound (Shore, 2005). It may serve to improve signal-tonoise ratios when attention is directed to a particular location (Oertel and Young, 2004; Shore, 2005).

\section{VGLUT1 mediates glutamate transport at ANF terminals}

Consistently with previous studies (Brown and Ledwith, 1990; Fekete et al., 1984; Shore and Moore, 1998), the present study demonstrated that infusion of FG into the labyrinth resulted in heavy labeling of eighth nerve axons and their terminals in the VCNm and DCN3. Large, endbulb-like ANF terminals endings surrounded large somata in VCNm. Small ANF boutons were located in the neuropil of both the VCNm and the DCN3.

The ANF endbulb synapse onto bushy cells provides a secure synaptic connection with the postsynaptic neurons and allows the relay of precise acoustic information. Colocalization of VGLUT1-ir with the FG-labeled AN endings around the somata of large neurons, as well as with small boutons in the neuropil, indicates that VGLUT1 mediates glutamate transport for the bushy cells as well as other cells that receive bouton-type endings on their dendrites, such as stellate or octopus cells (Fekete et al., 1984; Liberman, 1993; Tolbert and Morest, 1982). Small numbers of FG-labeled ANFs were observed in the shell region. These endings were thin $(\sim 1 \mu \mathrm{m})$, formed small en passant or terminal boutons, and were likely to be type II ANFs (Benson and Brown, 2004; Brown and Ledwith, 1990). These thin ANFs did not colocalize with either VGLUT1-ir or VGLUT2-ir. This finding is consistent with previous observations that glutaminase-like immunoreactivity is not seen in type II spiral ganglion cells (Altschuler et al., 1984), suggesting that a neurotransmitter other than glutamate is used by these cells.

\section{Possible roles of VGLUTs in the Sp5 and ANF terminals}

The slight structural difference between VGLUT1 and VGLUT2 may lead to different interactions with other proteins and, in turn, affect the vesicle filling and recycling of VGLUT isoforms at the nerve terminal (Fremeau et al., 2004b; Herzog et al., 2001). The finding that VGLUT1 mediates glutamate transport of the presumed

Fig. 6. Counts of labeled Sp5 terminal endings colocalized with VGLUT1-ir and VGLUT2-ir in different subdivisions of the CN ( $\mathrm{n}=$ 2). About $50 \%$ of MFs and $12 \%$ of small Sp5 boutons colabeled with VGLUT2. Significantly fewer $(2.5 \% \pm 1 \%)$ Sp5 MFs colabeled with VGLUT1 (paired $t$-test, $P<0.05$ ), and no small Sp5 boutons colabeled with VGLUT1; $23.6 \% \pm 1 \%$ of total Sp5 terminal endings (both MFs and small boutons) colabeled with VGLUT2, which is significantly greater than the endings colabeled with VGLUT1 $(2 \% \pm 0.1 \%$; paired $t$-test, $P<0.05)$. In contrast, many labeled ANFs $(79.5 \% \pm 7 \%$ of endbulb-like terminals and $43.0 \% \pm 3 \%$ of bead-like terminals) colocalized with VGLUT1 in the VCNm as well as in the DCN3 $(26.0 \% \pm$ $5 \%$ ). Neither ANF endbulb-like terminals nor small boutons was colocalized with VGLUT2. Error bars represent SEM. Asterisks indicate significant differences (see text). M, MFs; B, boutons; T, total (MFs and boutons); E, endbulb-like terminals. 


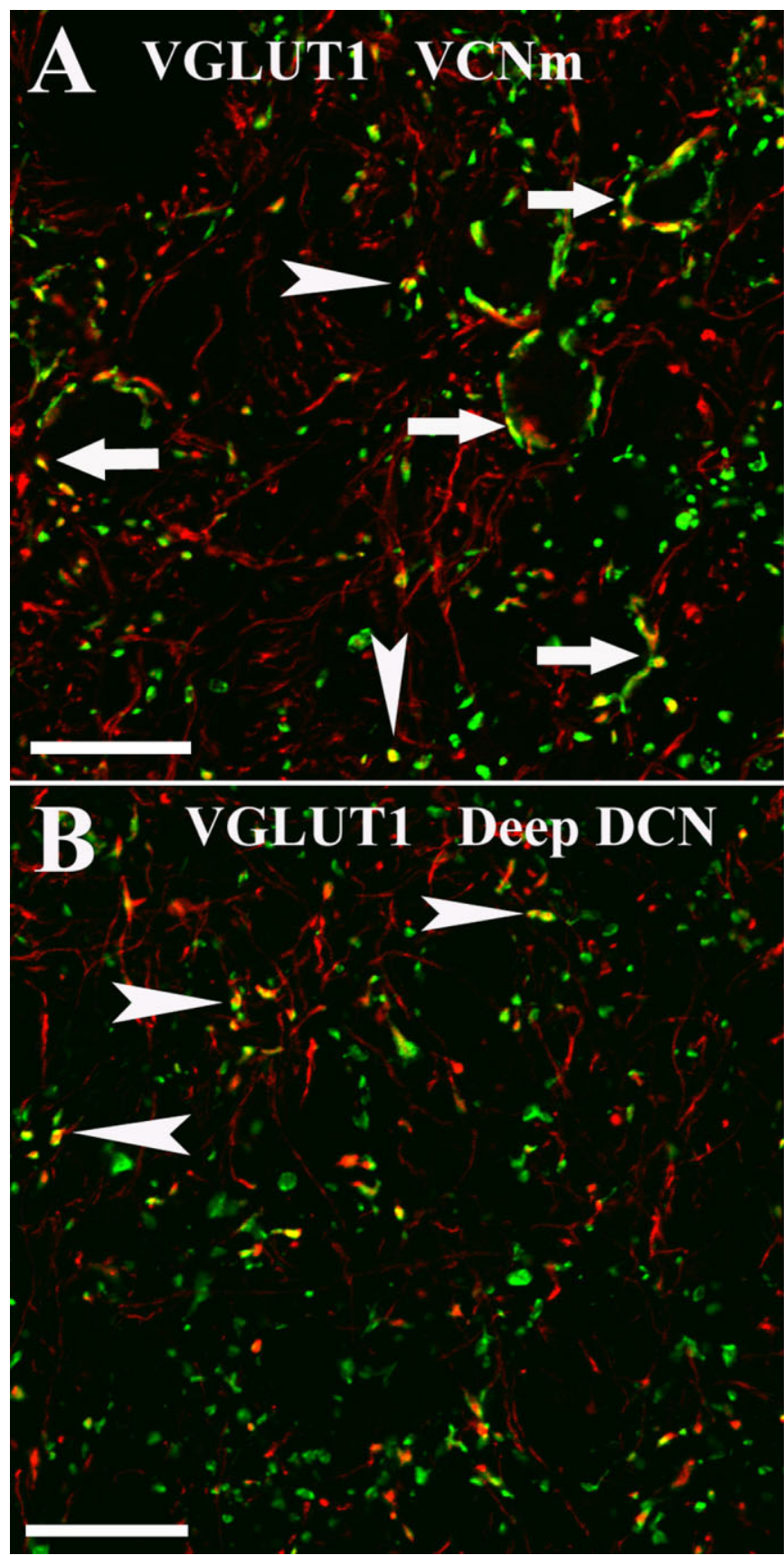

Fig. 7. High-magnification, $1-\mu \mathrm{m}$ confocal images $(\times 63)$ showing colocalization of AN terminal endings with VGLUT1-ir, but not VGLUT2-ir, in both VCNm and DCN3. Green, VGLUT-ir; red, FG filled AN fibers and endings; yellow, double-labeled terminals. All figures show a single, 1- $\mu \mathrm{m}$ confocal image. A,B: Colocalization of AN

type I ANFs in the CN suggests that VGLUT1 is likely involved in conveying the precise temporal information available in the acoustic signal. High temporal precision, for which fast packaging and recycling of glutamate is required, is accomplished by calyceal terminals in several major ascending pathways, including $\mathrm{CN}$ and superior olive complex (SOC). Strong VGLUT1 expression in the calyceal terminals in the SOC supports the concept that
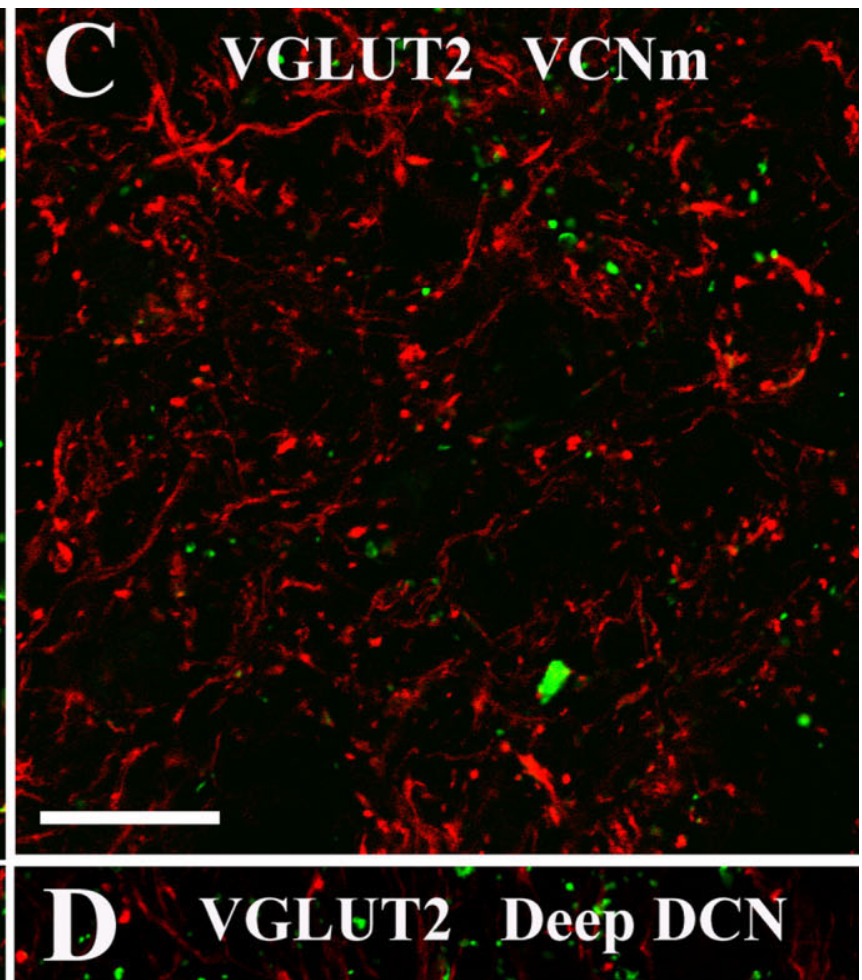

terminal endings with VGLUT1-ir in VCNm (A) and DCN3 (B). Both endbulb-like AN endings (arrows in A) and small boutons (arrowheads in A,B) colabeled with the VGLUT1. C,D: VGLUT2 did not label the AN endings in both VCNm (C) and DCN3 (D). Scale bars = $20 \mu \mathrm{m}$.

VGLUT1 is involved in conveying precise timing (Blaesse et al., 2005). However, it remains unclear whether the VGLUT1 in the presumed parallel fibers in the DCN1 is also associated with fast transmission.

In contrast to the rapid transmission of information between ANFs and magnocellular neurons in the CN, the transfer of information from the somatosensory system to the $\mathrm{CN}$ is likely to be either more modulatory or slower in 
nature. In the cerebellum, MFs are able to adjust synaptic strength via enhanced neurotransmitter release (Sola et al., 2004). Such a role in synaptic plasticity might also be expected in the DCN, with its cerebellar-like organization (Tzounopoulos et al., 2004). VGLUT2, which mediates glutamate transport of Sp5 MF endings, might therefore be associated with DCN synaptic plasticity.

The difference in recycling of the two isoforms at the nerve terminals has been previously demonstrated. In the stratum radiatum of the VGLUT1 knockout mouse, repetitive stimulation led to more rapid short-term depression with a slower recovery than in wild-type mice, suggesting that VGLUT2 is responsible for this plasticity. Thus, VGLUT1- and VGLUT2-mediated synapses originating from the same neuron may exhibit different forms of synaptic transmission. Clearly, more physiological evidence is needed to elucidate the functional significance of VGLUT isoforms in synaptic transmission (Fremeau et al., 2004a).

\section{ACKNOWLEDGMENTS}

We thank Sanford Bledsoe and Richard Altschuler for valuable comments on an earlier draft of the paper and Thankam S. Nair for expert technical assistance with Western blot analysis.

\section{LITERATURE CITED}

Abercrombie M. 1946. Estimation of nuclear population from microtome sections. Anat Rec 94:239-245.

Altschuler RA, Wenthold RJ, Schwartz AM, Haser WG, Curthoys NP Parakkal MH, Fex J. 1984. Immunocytochemical localization of glutaminase-like immunoreactivity in the auditory nerve. Brain Res 291:173-178.

Benson TE, Brown MC. 2004. Postsynaptic targets of type II auditory nerve fibers in the cochlear nucleus. J Assoc Res Otolaryngol 5:111-125.

Blaesse P, Ehrhardt S, Friauf E, Nothwang HG. 2005. Developmental pattern of three vesicular glutamate transporters in the rat superior olivary complex. Cell Tissue Res 320:33-50.

Brown MC, Ledwith JV 3rd. 1990. Projections of thin (type-II) and thick (type-I) auditory-nerve fibers into the cochlear nucleus of the mouse. Hear Res 49:105-118.

Davis KA, Miller RL, Young ED. 1996. Effects of somatosensory and parallel-fiber stimulation on neurons in dorsal cochlear nucleus. J Neurophysiol 76:3012-3024.

Fekete DM, Rouiller EM, Liberman MC, Ryugo DK. 1984. The central projections of intracellularly labeled auditory nerve fibers in cats. J Comp Neurol 229:432-450.

Fremeau RT Jr, Troyer MD, Pahner I, Nygaard GO, Tran CH, Reimer RJ, Bellocchio EE, Fortin D, Storm-Mathisen J, Edwards RH. 2001. The expression of vesicular glutamate transporters defines two classes of excitatory synapse. Neuron 31:247-260.

Fremeau RT Jr, Burman J, Qureshi T, Tran CH, Proctor J, Johnson J, Zhang H, Sulzer D, Copenhagen DR, Storm-Mathisen J, Reimer RJ, Chaudhry FA, Edwards RH. 2002. The identification of vesicular glutamate transporter 3 suggests novel modes of signaling by glutamate. Proc Natl Acad Sci U S A 99:14488-14493.

Fremeau RT Jr, Kam K, Qureshi T, Johnson J, Copenhagen DR, StormMathisen J, Chaudhry FA, Nicoll RA, Edwards RH. 2004a. Vesicular glutamate transporters 1 and 2 target to functionally distinct synaptic release sites. Science 304:1815-1819.

Fremeau RT Jr, Voglmaier S, Seal RP, Edwards RH. 2004b. VGLUTs define subsets of excitatory neurons and suggest novel roles for glutamate. Trends Neurosci 27:98-103.

Gras C, Herzog E, Bellenchi GC, Bernard V, Ravassard P, Pohl M, Gasnier B, Giros B, El Mestikawy S. 2002. A third vesicular glutamate transporter expressed by cholinergic and serotoninergic neurons. J Neurosci 22:5442-5451.

Haenggeli CA, Pongstaporn T, Doucet JR, Ryugo DK. 2005. Projections from the spinal trigeminal nucleus to the cochlear nucleus in the rat. J Comp Neurol 484:191-205.
Herzog E, Bellenchi GC, Gras C, Bernard V, Ravassard P, Bedet C, Gasnier B, Giros B, El Mestikawy S. 2001. The existence of a second vesicular glutamate transporter specifies subpopulations of glutamatergic neurons. J Neurosci 21:RC181.

Herzog E, Gilchrist J, Gras C, Muzerelle A, Ravassard P, Giros B, Gaspar P, El Mestikawy S. 2004. Localization of VGLUT3, the vesicular glutamate transporter type 3, in the rat brain. Neuroscience 123:983-1002.

Hioki H, Fujiyama F, Taki K, Tomioka R, Furuta T, Tamamaki N, Kaneko T. 2003. Differential distribution of vesicular glutamate transporters in the rat cerebellar cortex. Neuroscience 117:1-6.

Kaneko T, Fujiyama F, Hioki H. 2002. Immunohistochemical localization of candidates for vesicular glutamate transporters in the rat brain. J Comp Neurol 444:39-62.

Kanold PO, Young ED. 2001. Proprioceptive information from the pinna provides somatosensory input to cat dorsal cochlear nucleus. J Neurosci 21:7848-7858.

Levine RA. 1999. Somatic (craniocervical) tinnitus and the dorsal cochlear nucleus hypothesis. Am J Otolaryngol 20:351-362.

Li H, Mizuno N. 1997. Single neurons in the spinal trigeminal and dorsal column nuclei project to both the cochlear nucleus and the inferior colliculus by way of axon collaterals: a fluorescent retrograde doublelabeling study in the rat. Neurosci Res 29:135-142.

Liberman MC. 1993. Central projections of auditory nerve fibers of differing spontaneous rate, II: posteroventral and dorsal cochlear nuclei. J Comp Neurol 327:17-36.

Martin MR. 1985. Evidence for an excitatory amino acid as the transmitter of the auditory nerve in the in vitro mouse cochlear nucleus. Hear Res 20:215-220.

Nunzi MG, Russo M, Mugnaini E. 2003. Vesicular glutamate transporters VGLUT1 and VGLUT2 define two subsets of unipolar brush cells in organotypic cultures of mouse vestibulocerebellum. Neuroscience 122 : 359-371.

Oertel D, Young ED. 2004. What's a cerebellar circuit doing in the auditory system? Trends Neurosci 27:104-110.

Ohlrogge M, Doucet JR, Ryugo DK. 2001. Projections of the pontine nuclei to the cochlear nucleus in rats. J Comp Neurol 436:290-303.

Schweitzer L, Jensen KF, Janssen R. 1991. Glutamate neurotoxicity in rat auditory system: cochlear nuclear complex. Neurotoxicol Teratol 13: $189-193$.

Shore S. 2005. Multisensory integration in the dorsal cochlear nucleus: Responses to acoustic and trigeminal stimulation. Eur J Neurosci 21:3334-3348.

Shore S, Zhou J. 2006. Somatosensory influence on the cochlear nucleus and beyond. Hear Res 216-217/90-99.

Shore SE, Moore JK. 1998. Sources of input to the cochlear granule cell region in the guinea pig. Hear Res 116:33-42.

Shore SE, Vass Z, Wys NL, Altschuler RA. 2000. Trigeminal ganglion innervates the auditory brainstem. J Comp Neurol 419:271-285.

Sola E, Prestori F, Rossi P, Taglietti V, D'Angelo E. 2004. Increased neurotransmitter release during long-term potentiation at mossy fibregranule cell synapses in rat cerebellum. J Physiol 557:843-861.

Takamori S, Rhee JS, Rosenmund C, Jahn R. 2001. Identification of differentiation-associated brain-specific phosphate transporter as a second vesicular glutamate transporter (VGLUT2). J Neurosci 21:RC182.

Tolbert LP, Morest DK. 1982. The neuronal architecture of the anteroventral cochlear nucleus of the cat in the region of the cochlear nerve root: electron microscopy. Neuroscience 7:3053-3067.

Tzounopoulos T, Kim Y, Oertel D, Trussell LO. 2004. Cell-specific, spike timing-dependent plasticities in the dorsal cochlear nucleus. Nat Neurosci 7:719-725.

Weedman DL, Pongstaporn T, Ryugo DK. 1996. Ultrastructural study of the granule cell domain of the cochlear nucleus in rats: mossy fiber endings and their targets. J Comp Neurol 369:345-360.

Wright DD, Ryugo DK. 1996. Mossy fiber projections from the cuneate nucleus to the cochlear nucleus in the rat. J Comp Neurol 365:159-172.

Young ED, Nelken I, Conley RA. 1995. Somatosensory effects on neurons in dorsal cochlear nucleus. J Neurophysiol 73:743-765.

Zhou J, Shore S. 2004. Projections from the trigeminal nuclear complex to the cochlear nuclei: a retrograde and anterograde tracing study in the guinea pig. J Neurosci Res 78:901-907.

Zhou J, Shore S. 2006. Convergence of spinal trigeminal and cochlear nucleus projections in the inferior colliculus of the guinea pig. J Comp Neurol 495:100-112. 